\title{
Ice XII in its second regime of metastability
}

\author{
Michael Marek Koza \\ Fachbereich Physik, Universität Dortmund, D-44221 Dortmund, Germany \\ Helmut Schober, Thomas Hansen \\ Institut Laue-Langevin, F-38042 Grenoble, France \\ Albert Tölle \\ Fachbereich Physik, Universität Dortmund, D-44221 Dortmund, Germany \\ Franz Fujara \\ TU-Darmstadt, Hochschulstr. 6, D-64289 Darmstadt
}

(November 2, 2018)

\begin{abstract}
We present neutron powder diffraction results which give unambiguous evidence for the formation of the recently identified new crystalline ice phase [4], labeled ice XII, at completely different conditions. Ice XII is produced here by compressing hexagonal ice $\mathrm{I}_{\mathrm{h}}$ at $\mathrm{T}=77,100,140$ and $160 \mathrm{~K}$ up to $1.8 \mathrm{GPa}$. It can be maintained at ambient pressure in the temperature range $1.5<\mathrm{T}<135 \mathrm{~K}$. High resolution diffraction is carried out at $\mathrm{T}=1.5 \mathrm{~K}$ and ambient pressure on ice XII and accurate structural properties are obtained from Rietveld refinement. At $\mathrm{T}=140$ and $160 \mathrm{~K}$ additionally ice III/IX is formed. The increasing amount of ice III/IX with increasing temperature gives an upper limit of $\mathrm{T} \approx 150 \mathrm{~K}$ for the successful formation of ice XII with the presented procedure.
\end{abstract}

Although, water has been extensively studied both experimentally and theoretically it still rewards us with new and unexpected properties. This has been demonstrated recently by (i) the detection of polyamorphism [1] 3] and (ii) the discovery of a new crystalline phase (ice XII) [4.5. Having been observed in different regions of water's phase diagram the two phenomena were originally thought to be disconnected (Figure 11). The phenomenon of polyamorphism, i.e. the existence of two distinct amorphous phases, is still lacking a comprehensive understanding [6]. Although polyamorphism is equally observed in other substances particularly interesting explanations have been put forward for water. These ideas are based on computer simulations [7] and link polyamorphism to particularities of the supercooled liquid like phase segregation and a second critical point. Due to homogeneous crystallization supercooled water is experimentally inaccessible in the region where these phenomena are expected. The hypotheses must, therefore, be checked indirectly, e.g. by establishing the glassy character of the amorphous phases. The formation of the highdensity amorphous ice (HDA) - achieved by compressing crystalline hexagonal ice $\mathrm{I}_{\mathrm{h}}$ at temperatures below $150 \mathrm{~K}$ to pressures exceeding $1 \mathrm{GPa}$ (Fig. 1) - has received particular attention in this context [7 9].

So far, the formation of HDA from ice $\mathrm{I}_{\mathrm{h}}$ has been reported as a well-defined transition channel. The contamination of the amorphous samples by crystalline impurities has been granted little attention 10 12]. Only recently 13 - 15 strong experimental indications have become available which imply that all these contaminations correspond to ice XII. As ice XII was originally observed in a completely different region of water's phase diagram this shows that it is a rather prolific phase of water. Moreover, the co-production of ice XII has, as we will argue in the conclusions, far reaching implications for the $\mathrm{I}_{\mathrm{h}}$ to HDA transformation and, thus, on the origin of water's polyamorphism.

In this letter we show that the structure of ice XII produced at low temperatures is definitely identical with the phase characterized by Lobban et al. [4] at higher temperatures. Furthermore, we find that no continuous connection between the two regions of apparent metastability exists. And finally, we identify the conditions which define whether the compression of $\mathrm{I}_{\mathrm{h}}$ results in ice XII or HDA.

Our results are based on high-resolution neutron powder diffraction experiments on samples which are produced at various temperatures. All samples are treated in the following way. About $2.5 \mathrm{ml}$ of $\mathrm{D}_{2} \mathrm{O}$ (purity 99.9, resistivity $1 \mathrm{M} \Omega \mathrm{cm}$ ) is frozen to common hexagonal ice $\mathrm{I}_{\mathrm{h}}$ and cooled to $77 \mathrm{~K}$ in a piston-cylinder apparatus. The samples are heated to the desired temperatures, namely 77, 100, 140 and $160 \mathrm{~K}$ and tempered for about 30 minutes. Each sample is then compressed to a maximum nominal pressure of $1.8 \mathrm{GPa}$. The rate of compression is $1 \mathrm{GPa} / \mathrm{min}$ at $77 \mathrm{~K}$ and $0.5 \mathrm{GPa} / \mathrm{min}$ at all other temperatures. Once the maximum pressure is attained the samples are cooled back to $77 \mathrm{~K}$ and finally recovered from the pressure device in liquid nitrogen, where they are powdered by using a mortar and a pestle. No pressure changes which could be interpreted as signs of phase transitions are observed in the course of cooling under pressure. The sample temperature as measured at the bottom of the pressure cylinder apparatus does not increase by more than $10 \mathrm{~K}$ during the compression phase. 
The diffraction experiments are carried out on the high-resolution diffractometer D2B at the Institute-LaueLangevin in Grenoble, France [16]. A Vanadium sample holder (diameter $=7 \mathrm{~mm}$ ) and a standard cryostat are chosen as sample environment [16]. Two different experimental setups are applied. The accurate structure determination of ice XII is carried out at $1.5 \mathrm{~K}$ on the sample produced at $77 \mathrm{~K}$. For this measurement the horizontal incident beam divergence $\alpha_{1}$ is set to $10^{\prime}$ and the monochromator aperture (MA) to $10 \mathrm{~mm}$. The detector is moved using $\Delta \Theta=0.05^{\circ}$ steps. In this high resolution mode, the applied wavelength $\lambda=1.59427 \pm 0.00006 \AA$ and the instrumental resolution are determined from an independent measurement of a silicon powder sample. For good statistics data are collected over 15 hours. All other measurements are performed at $110 \mathrm{~K}$, with $\alpha_{1}=30^{\prime}$ and $\mathrm{MA}=50 \mathrm{~mm}, \Delta \Theta=0.05^{\circ}$ at the given wavelength. These lower resolution data are collected for 120 minutes (sample prepared at $77 \mathrm{~K}$ ) and 30 minutes (all other samples) which is sufficient for a structural identification of the samples.

Figure 2 displays the results of the high-resolution measurement at $1.5 \mathrm{~K}$. A Rietveld refinement [17] is performed without any constrains on the parameters using the program Fullprof 18 . Our refinement confirms that the structure of the observed ice phase is unambiguously that of ice XII [4, 13. This structure is characterized by twelve water molecules arranged in a tetragonal unit cell meeting the symmetry space group I $\overline{4} 2 \mathrm{~d}$. The refined unit cell parameters are $a=8.2816 \pm 0.0002 \AA$ and $b=4.0361 \pm 0.0001 \AA$ which result in a calculated microscopic density of $\rho=$ $1.4397 \pm 0.0003 \mathrm{~g} / \mathrm{cm}^{3}\left(\mathrm{D}_{2} \mathrm{O}\right)$. We present in Table $\mathrm{I}$ the refined fractional coordinates and thermal factors and in Table II the calculated intra- and intermolecular distances and bond angles. Taking into account the different preparational conditions which are used here and in reference $\mathbb{4}$ the cell constants, mass densities and atomic parameters are in good agreement with each other. Profile features in the diffraction pattern (Figure 2) which are not due to ice XII can be attributed to the sample environment and a slight contamination of the sample by some untransformed $\mathrm{I}_{\mathrm{h}}$ and simultaneously produced amorphous ice. This contamination is taken into account in the refinement by profile $\left(\mathrm{I}_{\mathrm{h}}\right.$, symmetry space group P6 $3 / \mathrm{mmc}$ ) and background (amorphous ice) matching [18,19]. The peak which due to the sample environment is excluded from the calculation. The formation of amorphous ice shows the ambivalent nature of the applied pressure induced transition. The final residuals of the refinement are $R_{w p}=3.48 \%$ and $R_{p}=2.69 \%$.

The diffraction results for samples prepared at different temperatures are compared in Figure 3 . The presence of ice XII can be clearly identified in all of the data, although, its diffraction pattern is obscured progressively by an additional contribution at 140 and $160 \mathrm{~K}$, respectively. To identify this additional contribution profile matching methods 18,19] are used for ice phases expected in this region of water's phase diagram. The most promising indexing scheme is given by the symmetry space group $\mathrm{P} 4_{1} 2_{1} 2$. This symmetry space group is uniquely inherent to ice III and ice IX, which differ in the degree of order in their proton sublattices 20 23]. As in ice XII, twelve water molecules inhabit the tetragonal unit cell of ice III/IX. Table III gives the unit cell constants and the corresponding mass densities of ice XII and ice III/IX as determined by the profile matching. The profile shape function, which is used in the matching procedure, is fully accounted for by the instrument resolution function as determined from the samples prepared at 77 and $100 \mathrm{~K}$ (Figure 3a,b). As a consequence only the cell constants (Table III) are freely adjustable for the phase mixtures. Typical residuals of the matching for the ice XII and ice III/IX mixtures are $R_{w p} \approx 6 \%$ and $R_{p} \approx 8 \%$. The inset of Figure 3 shows a diffraction pattern taken at $\lambda=3.00 \AA$ on the time-of-flight spectrometer IN5 of a sample prepared at $T \approx 165 \mathrm{~K}$. The absence of the $(220)$ peak of ice XII which should be well resolved at $2 \Theta \approx 70^{\circ}$ indicates that the content of ice XII is negligibly small. Thus, above $T>160 \mathrm{~K}$ solely ice III/IX is formed. The pressure induced formation of ice III/IX from $\mathrm{I}_{\mathrm{h}}$ at temperatures exceeding $140 \mathrm{~K}$ is in agreement with recent extensive studies [24,7,8]. Formation of ice $\mathrm{V}$ at temperatures exceeding $180 \mathrm{~K}$ [8] is not observed in any of our samples. This shows that despite the elevated compression rates used in our work the temperature of the sample during the compression stage stayed close to the cell temperature.

Summarizing, we have demonstrated that ice $I_{h}$ can be successfully compacted either into the high-density amorphous state (HDA) or into the crystalline phase ice XII. Both phases are produced by application of pressure exceeding $1.0 \mathrm{GPa}$ at temperatures below $150 \mathrm{~K}$ (Figure $\mathrm{B}$ ). Ice XII is a rather prolific feature in waters phase diagram. Its formation in two seemingly disconnected regions is unusual but not entirely surprising when we consider the fact that the formation is governed by dynamic variables like the compression rate, and, in addition, is in competition with other crystalline and amorphous phases. Ice XII can be recovered at ambient pressure and low temperature and can be stored at temperatures lower than $135 \mathrm{~K}$. At higher temperatures it starts to transform apparently to the metastable cubic phase [13:25] which itself is a precursor of the stable $\mathrm{I}_{\mathrm{h}}$ form. Production of different ice phases under seemingly identical conditions is not a new observation but related to the non-equilibrium character of the transitions. In the case of the metastable ice phases IV, XII and stable ice V (see Figure 11) phase discrimination is achieved via the cooling rates [4, 26 28]. In our example the compression rate seems to be the decisive control parameter. Having used higher compression rates than in previous experiments it was possible to obtain exclusive formation of ice XII. Given the higher density of recovered ice XII $\left(\rho \approx 1.44 \mathrm{~g} / \mathrm{cm}^{3}\right)$ in comparison to recovered HDA $\left(\rho \approx 1.30 \mathrm{~g} / \mathrm{cm}^{3}\right)$ and an anticipated kinetic character of the transitions such a dependence could be expected. The observation of explosive 
sound accompanied by abrupt loss of pressure indicates the development of shock waves during the compression which could play a major role in the transformation process.

The here established competition between crystallization and amorphization under close experimental conditions has to be properly acknowledged by all theoretical attempts trying to explain amorphous ice formation under pressure. Crystallization implies a reorganization and not merely a deformation of water's hydrogen bond network and, therefore, places more stringent conditions on the transition mechanism. A purely mechanical instability [9] as recently proposed for the formation of HDA is, to our opinion, insufficient to explain the ice XII formation. This holds unless the mechanical collapse is accompanied by high molecular mobility as for example in the case of a thermodynamic mechanical instability [29] or shock wave melting [30] which allow for crystalline reassembly. The requirement for high mobility, necessary for ice XII formation, equally explains the threshold value of around 1.0 GPa which corresponds to water's extrapolated melting line.

\section{ACKNOWLEDGMENTS}

Helpful discussions with W.F. Kuhs are greatfully acknowledged. This work is financially supported by the German Bundesministerium für Bildung und Forschung project No. 03-FU4DOR-5.

[1] O. Mishima, L.D. Calvert and E. Whalley, Nature, 310, 393, (1984).

[2] O. Mishima, L.D. Calvert and E. Whalley, Nature, 314, 76, (1985).

[3] O. Mishima, J. Chem. Phys., 98, 4878, (1993).

[4] C. Lobban, J. L. Finney and W. F. Kuhs, Nature, 391, 268, (1998).

[5] M. O'Keeffe, Nature, 392, 879, (1998).

[6] P.H. Poole, T. Grande, C.A. Angell and P.F. McMillan, Science, 275, 322, (1997).

[7] O.Mishima and H.E. Stanley, Nature, 396, 329, (1998).

[8] O. Mishima, Nature, 384, 546, (1996).

[9] J.S. Tse, D.D. Klug, C.A. Tulk, I. Swainson, E.C. Svensson, C.-K. Loong, V. Shpakov, V.R. Belosludov, R.V. Belosludov and Y. Kawazoe, Nature, 400, 647, (1999).

[10] L. Bosio, G.P. Johari and J. Teixeira, Phys. Rev. Lett., 56, 460, (1986).

[11] A. Bizid, L. Bosio, A. Defrain and M. Oumezzine, J. Chem. Phys., 87, 2225, (1987).

[12] M.-C. Bellissent-Funel, J. Teixeira and L. Bosio, J. Chem. Phys., 87, 2231, (1987).

[13] M. Koza, H. Schober, A. Tölle, F. Fujara, and T. Hansen, Nature, 397, 660, (1999).

[14] M.M. Koza, ILL Report No.ILL97KO10T, Report on the preparation and performance of time-of-flight experiments on the amorphous and polycrystalline solid $D_{2} O$, p. $32-36$, (1997).

[15] H. Schober, M. Koza, A. Tölle, F. Fujara, C.A. Angell and R. Böhmer, Physica B, 241-243, 897, (1998).

[16] The Yellow Book - Guide to neutron research facilities at the ILL, H.G. Büttner, E. Lelievre-Berna and F. Pinet, Institut Laue-Langevin, 1997.

[17] H.M. Rietveld, J. Appl. Cryst., 2, 65, (1969).

[18] J. Rodriguez-Carvajal, Rietveld, Profile Matching and Integrated Intensities Refinement of X-ray and/or Neutron Data, Laboratoire Leon Brillouin (CEA-CNRS).

[19] G.S. Pawley, J. Appl. Cryst., 14, 357, (1981).

[20] B. Kamb and A. Prakash, Acta Cryst., B 24, 1317, (1968).

[21] G.P. Arnold, R.G. Wenzel, S.W. Rabideau, N.G. Nereson and A.L. Bowman, J. Chem. Phys., 55, 589, (1971).

[22] S.J. La Placa and W.C. Hamilton, J. Chem. Phys., 58, 567, (1973).

[23] J.D. Londono, W.F. Kuhs and J.L. Finney, J. Chem. Phys., 98, 4878, (1993).

[24] A.K. Garg, phys. stat. sol. (a), 110, 467, (1988).

[25] W.F. Kuhs, D.V. Bliss and J.L. Finney, J. Physique, 48, C-1631, (1987).

[26] B. Kamb, A. Prakash and K. Knobler, Acta Cryst., 22, 706, (1967).

[27] H. Engelhardt and E. Whalley, J. Chem. Phys., 56, 2678, (1972).

[28] H. Engelhardt and B. Kamb, J. Chem. Phys., 75, 5887, (1981).

[29] V.P. Shpakov, J.S. Tse, V.R. Belosludov and R.V. Belosludov, J. Phys. Condens. Matter, 9, 5853, (1997).

[30] S.B. Kormer, Sov. Phys. Uspekhi, 11, 229, (1968). 
TABLE I. Fractional coordinates $x, y, z$, multiplicities $M$ of atomic positions and corresponding thermal factors $B_{i s o}$ of ice XII (prepared at $77 \mathrm{~K}$ and measured at $1.5 \mathrm{~K}$ and ambient pressure) as obtained from Rietveld refinement. Errors are given in standard Fullprof units.

\begin{tabular}{lccccc}
\hline \hline & $\mathrm{x}$ & $\mathrm{y}$ & $\mathrm{z}$ & $\mathrm{M}$ & $\mathrm{B}_{\text {iso }}$ \\
\hline $\mathrm{O}(1)$ & 0 & 0 & 0 & 4 & $0.437(39)$ \\
$\mathrm{O}(2)$ & $0.36464(20)$ & 0.25 & 0.125 & 8 & $0.905(28)$ \\
$\mathrm{D}(3)$ & $0.04044(32)$ & $0.08375(24)$ & $-0.14212(78)$ & 16 & $1.512(45)$ \\
$\mathrm{D}(4)$ & $0.28954(28)$ & $0.21988(31)$ & $0.29498(74)$ & $1.840(55)$ \\
$\mathrm{D}(5)$ & $0.42120(29)$ & $0.33618(26)$ & $0.23683(86)$ & $1.522(34)$ \\
& & & \\
\hline \hline
\end{tabular}

TABLE II. Intra- and intermolecular distances, bond lengths and their multiplicities for water molecules of ice XII determined at $1.5 \mathrm{~K}$ and ambient pressure.

\begin{tabular}{ccc}
\hline \hline Bond & Distance $(\AA)$ & Multiplicity \\
\hline $\mathrm{O}(1)-\mathrm{O}(2)$ & 2.80 & 6 \\
$\mathrm{O}(2)-\mathrm{O}(2)$ & 2.77 & 2 \\
$\mathrm{O}(1)-\mathrm{D}(3)$ & 0.96 & 4 \\
$\mathrm{O}(2)-\mathrm{D}(4)$ & 0.96 & 2 \\
$\mathrm{O}(2)-\mathrm{D}(5)$ & 0.97 & 2 \\
\hline & & Angle (o) \\
Bond angle & 107.0 & Multiplicity \\
\hline $\mathrm{O}(2)-\mathrm{O}(1)-\mathrm{O}(2)$ & 114.5 & 4 \\
$\mathrm{O}(2)-\mathrm{O}(1)-\mathrm{O}(2)$ & 93.5 & 2 \\
$\mathrm{O}(2)-\mathrm{O}(2)-\mathrm{O}(2)$ & 131.9 & 1 \\
$\mathrm{O}(2)-\mathrm{O}(2)-\mathrm{O}(1)$ & 83.1 & 2 \\
$\mathrm{O}(2)-\mathrm{O}(2)-\mathrm{O}(1)$ & 132.8 & 2 \\
$\mathrm{O}(1)-\mathrm{O}(2)-\mathrm{O}(1)$ & 110.9 & 1 \\
$\mathrm{D}(3)-\mathrm{O}(1)-\mathrm{D}(3)$ & 106.6 & 4 \\
$\mathrm{D}(3)-\mathrm{O}(1)-\mathrm{D}(3)$ & 99.1 & 2 \\
$\mathrm{D}(4)-\mathrm{O}(2)-\mathrm{D}(4)$ & 117.2 & 1 \\
$\mathrm{D}(4)-\mathrm{O}(2)-\mathrm{D}(5)$ & 99.9 & 2 \\
$\mathrm{D}(4)-\mathrm{O}(2)-\mathrm{D}(5)$ & 122.0 & 2 \\
$\mathrm{D}(5)-\mathrm{O}(2)-\mathrm{D}(5)$ & & 1 \\
\hline \hline
\end{tabular}

TABLE III. Cell constants a, c and mass density $\rho\left(\mathrm{D}_{2} \mathrm{O}\right)$ of ice XII and ice III/IX in samples prepared at given temperatures $\mathrm{T}$ and measured at $\mathrm{T}=110 \mathrm{~K}$ as determined by a profile matching method.

\begin{tabular}{|c|c|c|c|c|c|c|}
\hline & \multicolumn{3}{|c|}{ ice XII } & \multicolumn{3}{|c|}{ ice III/IX } \\
\hline $\mathrm{T}(\mathrm{K})$ & $\mathrm{a}(\AA)$ & $\mathrm{c}(\AA)$ & $\rho\left(\mathrm{g} / \mathrm{cm}^{3}\right)$ & $\mathrm{a}(\AA)$ & $\mathrm{c}(\AA)$ & $\rho\left(\mathrm{g} / \mathrm{cm}^{3}\right)$ \\
\hline \begin{tabular}{l|}
77 \\
\end{tabular} & 8.30 & 4.04 & 1.43 & - & - & - \\
\hline 100 & 8.30 & 4.04 & 1.43 & - & - & - \\
\hline 140 & 8.30 & 4.04 & 1.43 & 6.71 & 6.99 & 1.27 \\
\hline 160 & 8.30 & 4.03 & 1.44 & 6.71 & 6.99 & 1.27 \\
\hline
\end{tabular}


FIG. 1. The phase diagram of water. $(\bullet)$ represents the region in which ice XII is observed by Lobban et al. $(T=260 \mathrm{~K}$, $p=0.55 \mathrm{GPa}$ ). Please note that this region is fully encapsulated in the regime of the stable ice V. In addition, a third water phase namely the metastable ice IV can equally be formed in this region by employing an appropriate cooling process which is indicated by the vertical arrow. The insert sketches the pressure induced transition line of $\mathrm{I}_{\mathrm{h}}$ (thick solid line) as studied by $\mathrm{O}$. Mishima and followed up here. The light shaded area stresses the region in which ice XII is successfully formed in the present work. Horizontal arrows indicate that $\mathrm{I}_{\mathrm{h}}$ transforms by compression below $150 \mathrm{~K}$ to ice XII or HDA and above $150 \mathrm{~K}$ to ice III/IX. The dark shaded area displays the $150 \mathrm{~K}$ boundary studied by O. Mishima for HDA and equally observed for ice XII here.

FIG. 2. Neutron powder diffraction patterns of ice XII measured (bars) on the high-resolution diffractometer D2B $(\lambda=1.594$ $\AA$ ) at $1.5 \mathrm{~K}$ and ambient pressure and calculated (solid line) from a Rietveld refinement using the package Fullprof. Tick marks indicate the reflection positions of ice XII (upper panel) and untransformed $\mathrm{I}_{\mathrm{h}}$ (lower panel, relative amount of $0.8 \%$ ). The lower solid line gives the difference profile between the measured and calculated diffraction pattern. The arrow indicates a profile feature arising from the sample environment which is excluded from the refinement. The inset gives an enlarged plot of the data at $58^{\circ} \geq 2 \Theta \geq 150^{\circ}$.

FIG. 3. Diffraction patterns of samples prepared at different temperatures and measured with $\lambda=1.594 \AA$ at $\mathrm{T}=110 \mathrm{~K}$ and ambient pressure. The samples are produced at $77 \mathrm{~K}$ (a), $100 \mathrm{~K}$ (b), $140 \mathrm{~K}$ (c) and $160 \mathrm{~K}$ (d). ¿From profile matching calculations the patterns can be accounted for by ice XII (a, b) only and mixtures of ice XII and ice III/IX (c, d). Note that the amount of ice III/IX is progressively increasing as the temperature is raised. The inset shows a diffraction pattern taken at the time-of-flight spectrometer IN5 at $\lambda=3.00 \AA$ of a sample prepared at $T \approx 165 \mathrm{~K}$. 


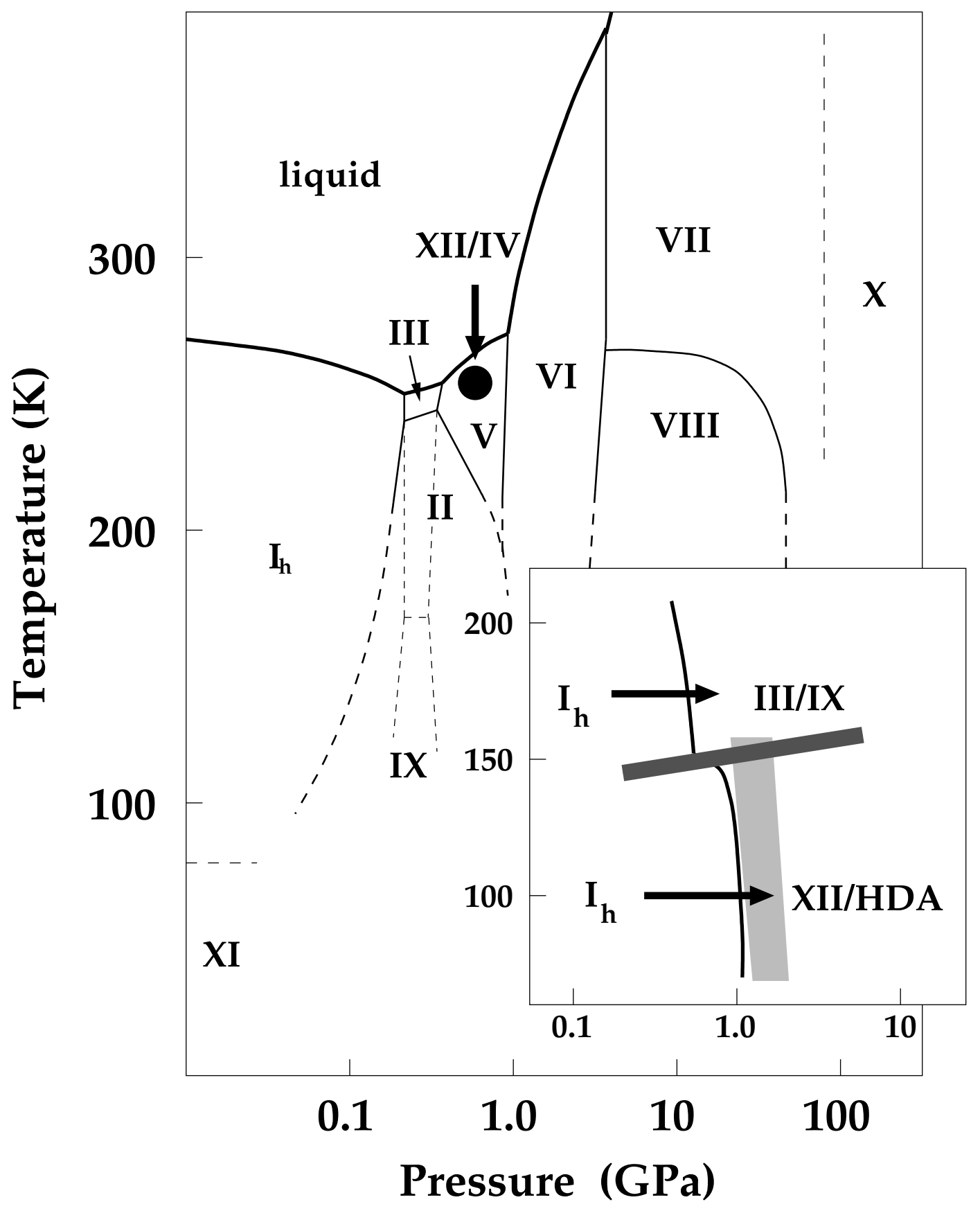

Koza et al. Fig. 1 


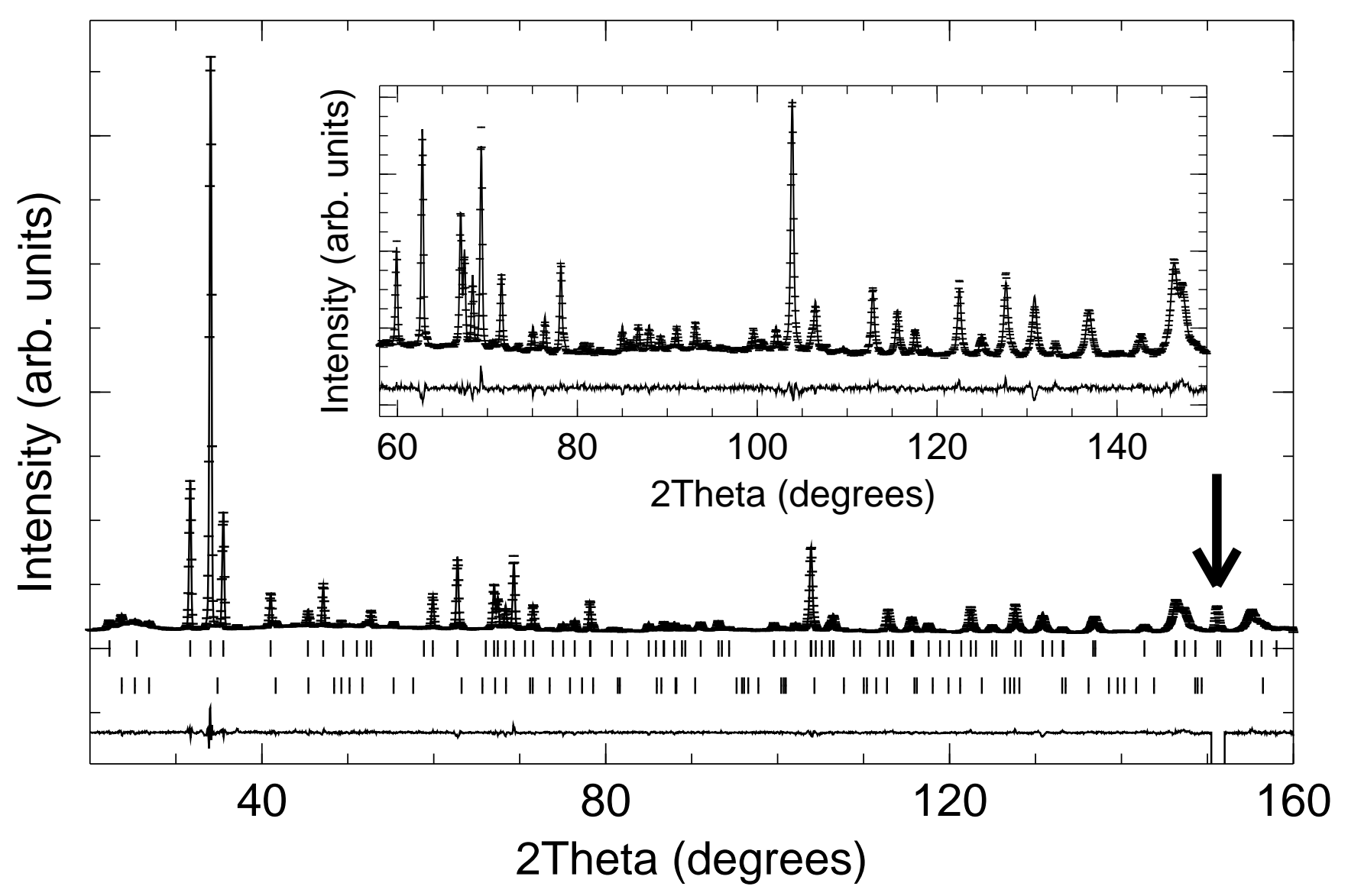

Koza et al. Figure 2 


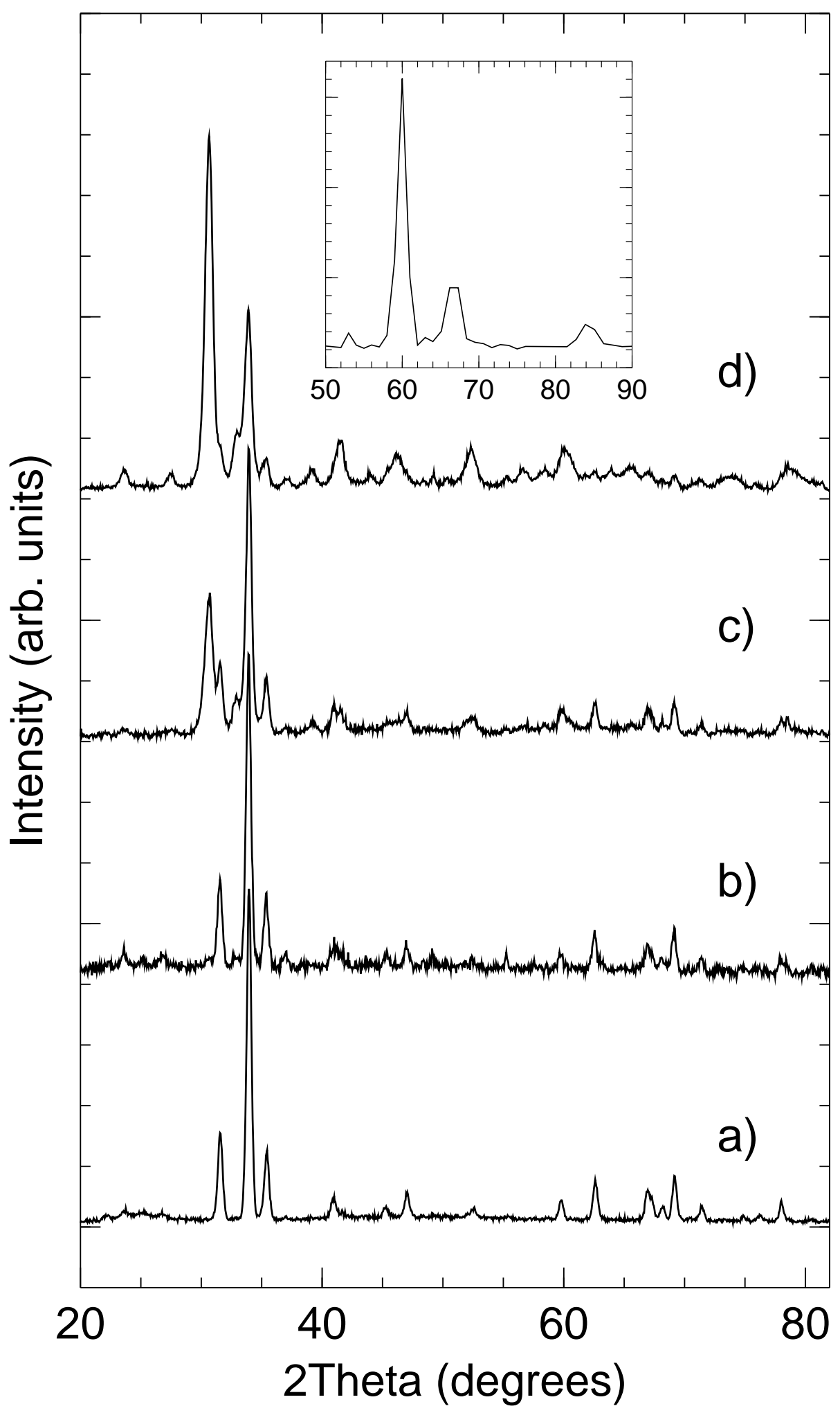

Koza et al. Fig. 3 